\title{
Cryo Electron Microscopy at the Bio21 Ian Holmes Imaging Center and in the wider Australian microscopy community
}

\author{
Eric Hanssen
}

The University of Melbourne, United States

In the last 5 years the Commonwealth of Australia and its scientific community have invested very heavily in electron cryo microscopy (cryoEM) to enhance or develop nodes of knowledge around the country. Most of these facilities are open access and allow the wide structural and cell biology community to access techniques that are rapidly becoming vital in many research project but too costly to implement in individual laboratory in the context of the Australian funding landscape. Here we will present an example of one of the newly re-developed facility at the University of Melbourne, the Bio21 Ian Holmes Imaging Center (IHIC). We will also give a wide overview of cryoEM equipment access around the country (Figure 1) and the different links that are in place to help researchers access the technology.

The IHIC (Figure 2) has been redeveloped over the last five years from a local high end "old school" microscopy unit to one of the Australian flagship 3D and cryoEM center to cater for a growing number of interested cell and structural biologists in the 10,000 scientists strong Parkville Biomedical precinct. The development of a brand new building to house the IHIC has allowed for investment in otherwise impossible to house equipment and delivered a building that is far from the historical basement location of electron microscopy facilities. The IHIC now houses five cryoTEMs, three of which are equipped with direct detectors (Glacios, Arctica G2, Krios G4), as well as all the sample preparation equipment. Data is acquired either by a facility staff or an experienced user and processes on-the-fly using cryoSPARC or Relion depending on user request.

The IHIC as most other facilities around the country operate as open access. However, there are very wide variation in terms of training and hands-on in different locations around the country. Access requirement at the IHIC and the different side of the access spectrum around the country will be discussed. One of the bottle neck in cryoEM is access to high performance computing and Australia like most other worldwide locations has different solution depending on the location of the facility used and the origin of the user. This is currently being discussed at a national level in order to implement an overarching solution allowing users to access/analyses their data in a timely manner irrespectively of their origin.

Acknowledgements: Thanks to Matthias Floettenmeyer (University of Queensland), Filip Braet (University of Sydney), Nick Ariotti (University of New South Wales), Georg Ramm (Monash University), Ashley Slattery (University of Adelaide), Melanie Rug (Australia National University), Peta Clode (University of Western Australia), James Bower (University of Wollongong) for data from their respective centers. 


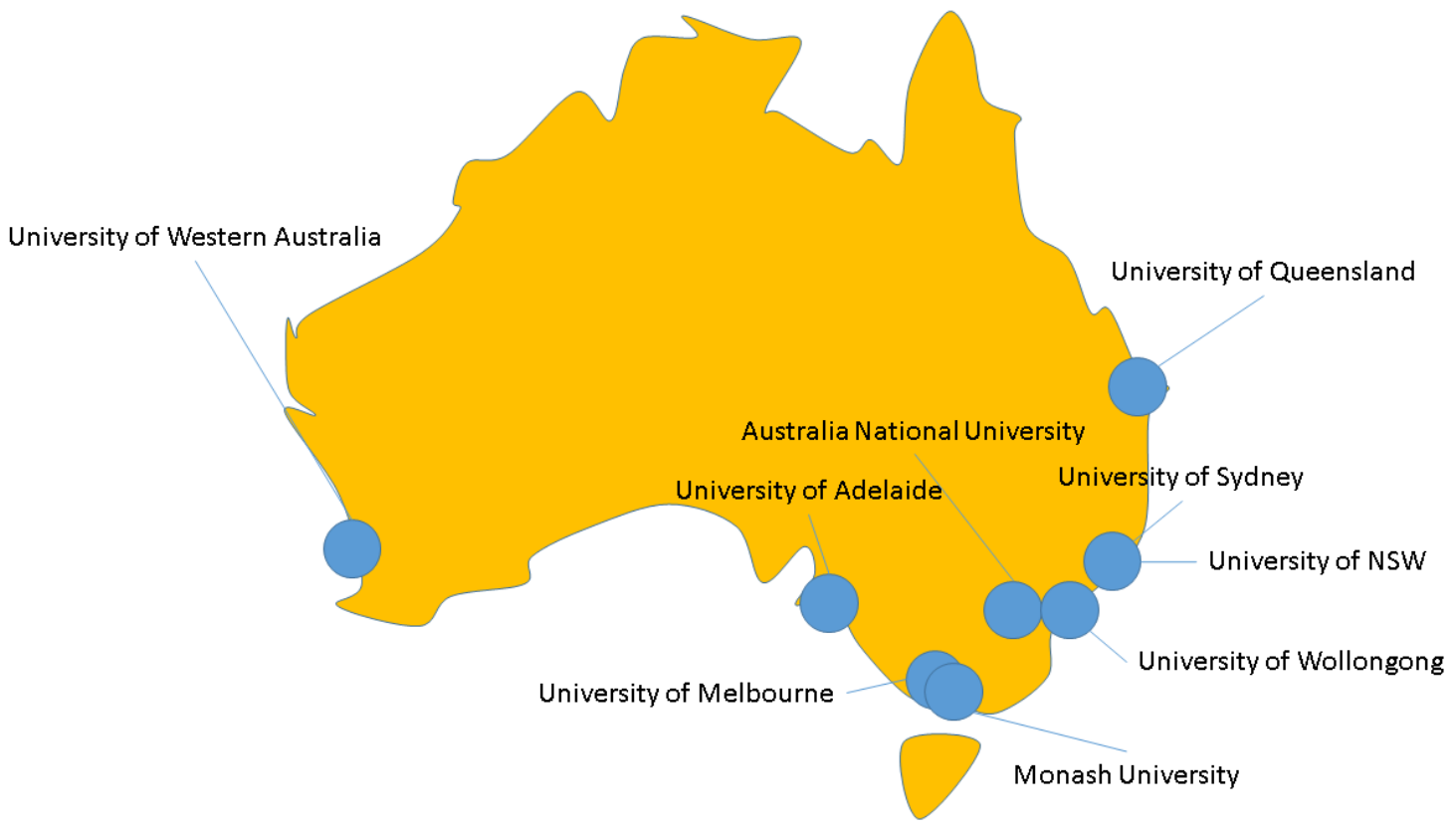

Figure 1. Location of current cryoTEMs with direct detectors

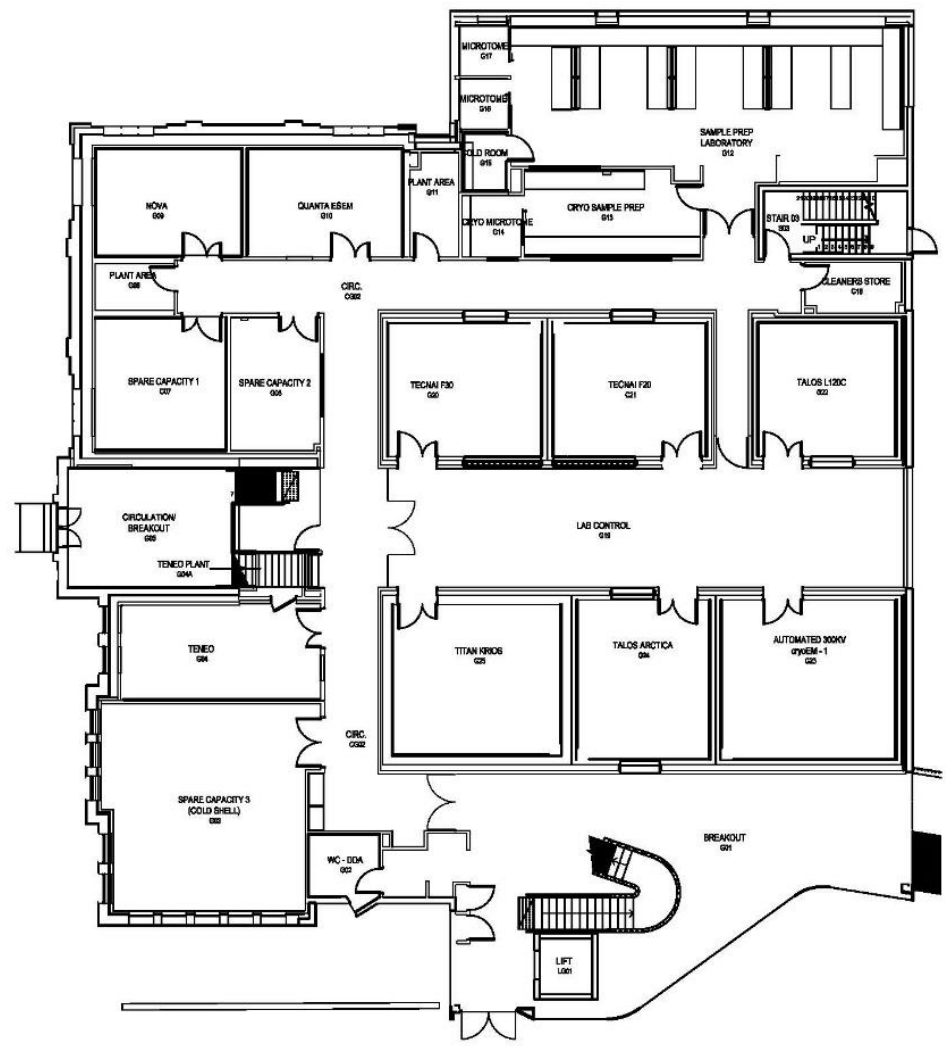

Figure 2. Floor plan of the Bio21 Ian Holmes Imaging Center 